\title{
UNIVERSAL OPERATORS AND INVARIANT SUBSPACES
}

\section{S. R. CARADUS}

For any Banach space $X$, let $B(X)$ denote the space of continuous endomorphisms of $X$. An operator $U$ in $B(X)$ will be called universal if, given any $T$ in $B(X)$, then some nonzero multiple of $T$ is similar to a part of $U$ i.e. there exists $\lambda \in C, \lambda \neq 0$, a closed subspace $X_{0}$ of $X$ such that $U X_{0} \subseteq X_{0}$ and a linear homeomorphism $\phi$ of $X$ onto $X_{0}$ such that $\lambda T=\phi^{-1}\left(U \mid X_{0}\right) \phi$. The first example of a universal operator (or model) was constructed by G.-C. Rota [1] for the Hilbert space case. In that instance, $U$ is (unitarily equivalent to) the direct sum of countably many copies of the reverse shift $\left(\xi_{1}, \xi_{2}, \xi_{3}, \cdots\right) \rightarrow$ $\left(\xi_{2}, \xi_{3}, \xi_{4}, \cdots\right)$. Such a direct sum obviously defines an operator whose nullspace is infinite-dimensional and whose range is the whole space. In this note, we show that all such operators are universal (when $X$ is a separable Hilbert space) and that, with rather obvious modifications, the arguments extend to arbitrary Banach spaces.

TheOREM. Let $X$ denote a separable Hilbert space and let $U$ belong to $B(X)$. If $U$ has the following properties:

(i) the nullspace $N(U)$ is infinite-dimensional,

(ii) the range space $R(U)$ is the space $X$, then $U$ is universal.

Proof. We begin by constructing operators, $V, W$ in $B(X)$ such that $U V=I, U W=0, N(W)=(0), R(W)$ is closed and $R(W) \perp R(V)$. To do this, we write $\tilde{U}$ for the restriction of $U$ to $N(U)^{\perp}$ and define $V=\tilde{U}^{-1}$. We then take an orthonormal basis $\left\{e_{n}\right\}$ for $X$ and an orthonormal basis $\left\{e_{n}^{\prime}\right\}$ for $N(U)$ and define $W e_{n}=e_{n}^{\prime}$. That $V$ and $W$ have the required properties is obvious. Now let $T$ be any operator in $B(X)$. Choose $\lambda$ so that $|\lambda|\|T\|\|V\|<1$ and define $\phi$ $=\sum_{k=1}^{\infty} \lambda^{k} V^{k} W T^{k}$, observing that, by choice of $\lambda$, this series converges in $B(X)$. It is also evident that

$$
U \phi=\lambda \phi T
$$

and

$$
\phi=\lambda V \phi T+W .
$$

We can now deduce from (2) that $\phi$ is a linear homeomorphism. For suppose $\phi(x)=0$. Then since $R(W) \perp R(V)$, it is evident that $V \phi T x$ $=W x=0$. But $W$ is invertible so $x=0$. Secondly, to show $R(\phi)$ is

Received by the editors April 24, 1969. 
closed, consider $\phi\left(x_{n}\right) \rightarrow y$. Then from (2), we have $\lambda V \phi T x_{n}+W x_{n} \rightarrow y$. Hence $W x_{n} \rightarrow P y$ where $P$ is the orthogonal projection onto $R(W)$. Since $R(W)$ is closed, there exists $x$ such that $W x_{n} \rightarrow W x$ and hence $x_{n} \rightarrow x$. Thus $\phi\left(x_{n}\right) \rightarrow \phi(x)=y$. Finally, from (1), $R(\phi)$ is $U$ invariant and the result follows.

Extension to THE General case. It is evident that the above proof is valid whenever we can perform the construction of $V$ and $W$ such that there is a continuous projection onto $R(W)$. In the case of arbitrary Banach space, the theorem is valid if we replace (i) by $(\mathrm{i})^{\prime}: N(U)$ is a complemented subspace containing a subspace which is linearly homeomorphic to $X$.

For suppose (i)' and (ii) hold and $X_{0}$ is a closed complement of $N(U)$. Let $\tilde{U}=U \mid X_{0}$ and define $V=\tilde{U}^{-1}$. Then for $W$, take the linear homeomorphism with range in $N(U)$ whose existence is asserted by (i)'.

Applications. Let $X$ be a separable Hilbert space. For any $T \in B(X)$, write $\mathscr{g}(T)$ to denote the lattice of closed invariant subspaces of $T$. If $g(T) \neq\{0, X\}$, we call $g(T)$ nontrivial.

(1) Either $g(T)$ is nontrivial for every $T$ or for every universal operator $U, g(U)$ has an infinite-dimensional atom.

(2) If $U$ has properties (i) and (ii), then there is a closed subspace $X_{0}$ such that $U X_{0}=X_{0}$ and $X_{0} \cap N(U)=(0)$.

For take $T$ which is invertible in $B(X)$. Then from equation (1), $U R(\phi)=R(\phi)$ and $N(U) \cap R(\phi)=(0)$.

(3) Suppose $U$ has properties (i) and (ii). Then $\mathscr{g}(U)$ contains a countable family of mutually disjoint nonzero subspaces $X_{n}$ such that $X_{n} \cap N(U)=(0)$.

For let $\left\{W_{n}\right\}$ be a sequence of operators with $R\left(W_{n}\right) \cap R\left(W_{m}\right)$ $=(0),(m \neq n), R\left(W_{n}\right)$ closed, $N\left(W_{n}\right)=(0)$ and $R\left(W_{n}\right) \subseteq N(U)$. Such a family can be constructed by the method used for constructing $W$ in the proof of the theorem. Take $V$ as in the proof and any $T$ with $\|T\|<\|V\|^{-1}$ and $N(T)=(0)$. Each $W_{n}$ defines a linear homeomorphism $\phi_{n}=\sum_{k=0}^{\infty} V^{k} W_{n} T^{k}$. Moreover, if $m \neq n, R\left(\phi_{n}\right) \cap R\left(\phi_{m}\right)=(0)$. For suppose $\phi_{n}(X)=\phi_{m}(y)$ then $W_{n} x+\sum_{k=1}^{\infty} V^{k} W_{n} T^{k} x=W_{m} y$ $+\sum_{k=1}^{\infty} V^{k} W_{m} T^{k} y$ so that $W_{n} x-W_{m} y \in N(U) \cap R(V)$. Thus $W_{n} x$ $=W_{m} y=0$ and therefore $x=y=0$.

\section{BIBLIOGRAPHY}

1. G.-C. Rota, On models for linear operators, Comm. Pure Appl. Math. 13 (1960), 469-472.

Australian National University and Queen's University at Kingston 